\title{
Friction between a temperature dependent viscoelastic body and a rough surface
}

\author{
Rainer HEISE* \\ Institute for Mechanics, Technische Universität Berlin, Straße des 17. Juni 135, Berlin D-10623, Germany \\ Received: 04 August 2015 / Revised: 30 November 2015 / Accepted: 01 January 2016 \\ (C) The author(s) 2016. This article is published with open access at Springerlink.com
}

\begin{abstract}
In this study, we investigate the friction between a one-dimensional elastomer and a one-dimensional rigid randomly rough surface. Special emphasis is laid on the temperature dependence of the elastomer and its effect on the frictional behavior of the contact. The elastomer is modeled as a Kelvin body in a one-dimensional substitute model in the spirit of the method of dimensionality reduction. The randomly rough surface is a self-affine one-dimensional fractal. We provide a short discussion of a conical indenter pressed in a displacement controlled process into an elastomer. These analytical considerations are taken as a basis for the treatment of the randomly rough counter surface in contact to an elastomer with and without temperature dependent viscosity. We identify dimensionless quantities describing this process, introduce a thermal length scale, and give estimates for the coefficient of friction as function of velocity, indentation and thermal quantities.
\end{abstract}

Keywords: elastomer friction; temperature dependence; coefficient of friction

\section{Introduction}

The roughness of interfaces seems to be the main source of friction according to the groundbreaking work of Bowden and Tabor [1]. Greenwood and Tabor [2] explained the behavior of polymers in frictional processes as deformation losses in the material. Experiments by Grosch [3] supported these ideas looking at friction between elastomers and hard specimens with controlled roughness. In the following years, the aspects of rheology [4] and surface roughness $[5,6]$ in rubber friction were investigated. The notion of the coefficient of friction is mostly used in studies in the field. Hereby, Amontons' laws are implicitly presumed to be valid: The force of friction is proportional to the normal force and hence the coefficient of friction is independent of the normal force $[7,8]$. This is a widely accepted relation, which is a rather simplified picture of real frictional systems. It is well established that both the static and the dynamic

* Corresponding author: Rainer HEISE.

E-mail: Rainer.Heise@tu-berlin.de coefficient of friction can vary by a factor of four depending on geometrical and loading conditions of the tribological system under investigation [9]. Schallamach [10] conducted experiments in polymer friction. Recently, deviations from Amontons' law were investigated [11, 12]. They may have their origin in macroscopic interfacial dynamics [13-15] or in the contact mechanics of rough surfaces. In this note, we explore the thermal behavior of elastomers due to frictional interaction under circumstances when the applicability of Amontons' laws is at stake. Some fundamental understanding of the frictional answer of a viscoelastic body is learned from a simplistic model: (i) the polymer is modeled as a Kelvin body, which is completely defined by a constant elastic modulus and a thermally activated viscosity, (ii) the undeformed elastomer surface is flat and experiences no friction on microscopic scale, (iii) the counter body is rigid, thermally insulated and has a randomly rough, selfaffine fractal surface, (iv) capillary and adhesive effects [16] are not considered, and (v) the features are investigated in a one-dimensional substitute model. 
Even though these requirements simplify the situation greatly we still observe a non-trivial frictional and thermodynamic behavior of the elastomer. We do not claim a direct one-to-one correspondence to a complete three-dimensional analysis but see a broad utilization of our results on one-dimensional grounds if the rules put forward in the method of dimensionality reduction (MDR) [17-21] are obeyed. The MDR maps threedimensional frictional problems onto one-dimensional ones. Li et al. [22] deal with friction of an elastomer surface with a rough surface at constant temperature. Dimaki and Popov [23] consider a smooth cone indenting into a polymer with temperature dependent viscosity. In Ref. [24], a randomly rough rigid surface is brought into contact with an elastomer of temperature dependent viscosity in a force controlled process.

This study is organized as follows. After a short introduction to rough surfaces in Section 2, the polymer model considered is presented and the contact criterion is established. Power dissipation and temperature dependence are then introduced. Section 3 provides a discussion of a single asperity contact, of the extension to rough multi-asperity surfaces, and of temperature dependence. A thermal length scale is identified. Finally, in Section 4 conclusions are drawn and an outlook is presented.

\section{Modeling}

\subsection{Self-affine isotropic fractal surfaces}

For the exploration of frictional behavior in the concept of MDR, two different steps have to be taken. On the one hand, the geometry of the contacting surfaces has to be transferred into a one-dimensional substitute model. On the other hand, physical observables in the contact have to be calculated in the model. Let us introduce a specific class of surfaces that we use for our model building.

Certain statistical key values may describe an arbitrary surface. Here, we name the root mean square (RMS) roughness $h \equiv \sqrt{\left\langle h^{2}\right\rangle}$ and the RMS gradient $\nabla h \equiv \sqrt{\left\langle(\nabla h)^{2}\right\rangle}$, where $\langle\cdots\rangle$ denotes the ensemble average over several realizations of the system. For a certain class of surfaces, so called randomly rough surfaces, there exists a close relation of the above quantities to the autocorrelation function (power spectrum) $C$, which completely characterizes the surface. Many natural surfaces are known to exhibit the property of fractality, at least in some range of wave vectors. In a one-dimensional substitute model, the roughness and the slope, i.e., the moments of the autocorrelation function, can be held constant at the values of an original two-dimensional surface [20]

$$
\begin{aligned}
\left\langle h^{2}\right\rangle_{1 \mathrm{~d}} & =\int_{-\infty}^{\infty} \mathrm{d} q C_{1 \mathrm{~d}}(q), \\
\left\langle(\nabla h)^{2}\right\rangle_{1 \mathrm{~d}} & =\int_{-\infty}^{\infty} \mathrm{d} q q^{2} C_{1 \mathrm{~d}}(q)
\end{aligned}
$$

where $q$ denotes the wave number.

The considered surfaces are fractal and self-affine surfaces meaning that the surface looks about the same as the resolution is increased or decreased. No natural length scale is to be found in this kind of surfaces. The Hurst exponent $H$ is another quantity to characterize fractal surfaces. Under a rescaling of the spatial coordinate $x \rightarrow \kappa x$ the height coordinate $h$ changes to $\kappa^{H} h$.

A one-dimensional self-affine isotropic surface $h$ is described by a power spectrum [20]

$$
C_{1 \mathrm{~d}}(q)=\hat{c}_{0} q_{f}^{-3}\left(\frac{q}{q_{f}}\right)^{-2 H-1} \quad q_{i} \leqslant q \leqslant q_{f}
$$

with dimensionless strength $\hat{c}_{0}$ and cut-off wave vectors $q_{i}$ and $q_{f}$. The RMS roughness and the RMS slope of the surface are computed as

$$
\begin{aligned}
\left\langle h^{2}\right\rangle & =\frac{\hat{c}_{0} q_{f}^{-2}}{H}\left(\left(\frac{q_{f}}{q_{i}}\right)^{2 H}-1\right), \\
\left\langle(\nabla h)^{2}\right\rangle & =\frac{\hat{c}_{0}}{1-H}\left(1-\left(\frac{q_{i}}{q_{f}}\right)^{2-2 H}\right)
\end{aligned}
$$

Eliminating the strength $\hat{c}_{0}$, the gradient of the surface is given by

$$
\left\langle(\nabla h)^{2}\right\rangle=q_{f}^{2} h^{2} \frac{H}{1-H} \frac{1-\left(\frac{q_{i}}{q_{f}}\right)^{2-2 H}}{\left(\frac{q_{f}}{q_{i}}\right)^{2 H}-1}
$$

Keeping in mind that the scale are well separated $q_{f} / q_{i} \gg 1$, 


$$
\left\langle(\nabla h)^{2}\right\rangle=q_{f}^{2} h^{2} \frac{H}{1-H}\left(\frac{q_{i}}{q_{f}}\right)^{2 H} \quad 0<H<1
$$

The slope interpolates between the values for longest respectively shortest wave vector for Hurst exponents in the range 0 to 1 according to a power law with $H$ as exponent.

\subsection{Discrete realization of rough surface}

For the generation of a randomly rough surface with the desired properties, we fall back on the Fourier transform:

$$
h_{1 \mathrm{~d}}=\frac{1}{2 \pi} \int_{-\infty}^{\infty} \mathrm{d} q B_{1 \mathrm{~d}}(q) \exp [i \phi(x)]
$$

The one-dimensional Fourier coefficients of the surface are proportional to the square root of the power spectrum [20]:

$$
B_{1 \mathrm{~d}}=\sqrt{\frac{2 \pi}{L} C_{1 \mathrm{~d}}}
$$

The randomness of the different realizations of the surface is assured by a uniformly distributed random phase $\phi$. For a discrete realization, the maximal and minimal wave vectors depend on the spatial step $\mathrm{d} x$ and the system length $L$ :

$$
q_{f}=\pi / \mathrm{d} x, \quad q_{i}=2 \pi / L
$$

The one-dimensional surface is generated as the inverse discrete Fourier transform (DFT). In the following we use a one-dimensional rigid surface $z_{0}$ expressed as

$$
z_{0}=\sum_{k=1}^{N} B_{1 \mathrm{~d}}(k) \exp \left[\frac{2 \pi i}{N}(j-1)(k-1)\right]
$$

\subsection{Elastomer}

After having described the generation of the onedimensional substitute surface we now turn to the modeling of the elastomer and the contact itself. In the considered model, two one-dimensional substitute surfaces $z$ and $u$ move relative to each other with constant velocity $v_{0}$. The surfaces are discretized and the individual sites are labeled by $i=1, \cdots, N$. In every site $i$, a spring damper combination models the elastomer. This combination is a Kelvin body as shown in Fig. 1.

It consists of a spring with stiffness [20]

$$
k=4 G \mathrm{~d} x
$$

which is coupled in parallel with a damper with damping constant [20]

$$
\theta=4 \eta \mathrm{d} x
$$

for incompressible media with Poisson number $v=1 / 2$. The material of the original polymer is described by an elastic modulus $G$ and a viscosity $\eta$. The factors of four originate from the employment of a one-dimensional substitute model [20, 25]. The onedimensional model, the elastomer surface is considered to be a chain of non-interacting Kelvin elements (Fig. 2).

For the viscoelastic model, the equation of motion is easily found for the Kelvin element at every site

$$
F_{\text {ext }}=-k\left(u-u_{0}\right)-\theta \dot{u}
$$

for some reference coordinate $u_{0}$. In terms of discretized variables, the force exerted at each site in contact is

$$
f_{i}=-k\left(u_{i}+\tau \frac{u_{i}-u_{i+1}}{\mathrm{~d} t}\right)
$$

where $\tau=\eta / \mathrm{G}$ is the relaxation time of the Kelvin element. The spring experiences a force according to

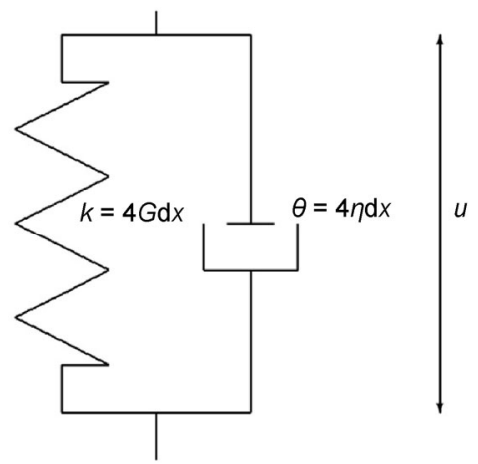

Fig. 1 Kelvin element.

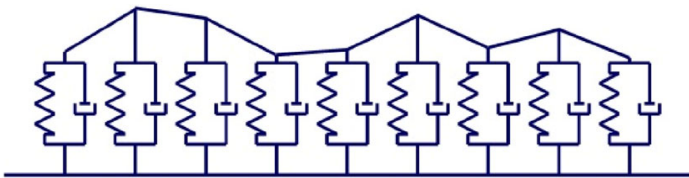

Fig. 2 Chain of Kelvin elements. 
the deviation from the undisturbed soft surface $u_{0 i}=0$ while the damper exerts a force that is proportional to the rate of change in the deformed surface. We identify $\theta v_{0} /(k \mathrm{~d} x)=\tau / \mathrm{d} t=\eta v_{0} /(G \mathrm{~d} x)$ as the ratio between the time scale of motion $\mathrm{d} t=\mathrm{d} x / v_{0}$ and the time scale of the viscoelastic material (relaxation) $\tau=\theta / k=\eta / G$. Between the elements of the surfaces, a force acts according to viscoelastic model Eq. (13) if it is in contact. The force is set to zero if surfaces are not in contact. Negative forces are not considered since this would correspond to adhesive effects which we exclude from our study.

Initially, the rigid surface described by coordinates $z_{i}$ is generated with a certain given RMS roughness $h$, system length $L$, spacing $\mathrm{d} x$ and Hurst exponent $H$. This fixes also the RMS gradient

$$
\nabla z=\sqrt{1 / N \sum_{i=1}^{N}\left(\left(z_{i+1}-z_{i}\right) / \mathrm{d} x\right)^{2}}
$$

and the cutoff wave vectors $q_{i}$ and $q_{f}$. Note that the mean of $z_{0}$ is zero by construction. The moving rigid surface is pressed into the deformable surface in such a way that a given indentation $d$ is sustained. The soft deformed lower surface is now described by coordinate $u_{\mathrm{i}}$.

The situation is viewed as a stationary system so that all transient features have disappeared. The side step $\mathrm{d} x$ is fixed as well as time step $\mathrm{d} t$ throughout this study. In particular, this means that spatial derivatives are linked to time derivatives via

$$
\frac{\mathrm{d}}{\mathrm{d} t}=v_{0} \frac{\mathrm{d}}{\mathrm{d} x}
$$

Imagine we move along with the rough surface at constant speed $v_{0}$ to the right. One point of the surface is transferred to a new position some distance ahead. At this new position, the coordinate of the relaxing interface $u_{\mathrm{i}}$ is calculated according to Eq. (13) without external force. In a continuous description, this leads to a simple ordinary differential equation

$$
0=u-u_{0}+\tau \dot{u}
$$

which is solved by

$$
u(t)=u(0) \exp \left[-\frac{t}{\tau}\right]
$$

after some time $t$ for an undisturbed surface at $u_{0}=0$ for some constant $u(0)$.

In the discretized case, the solution relates one site to the coordinate one step further to the right

$$
u_{i}=u_{i+1} \exp \left[-\frac{\mathrm{d} t}{\tau}\right]
$$

This solution corresponds to a free evolution and relaxation of the elastomer.

\subsection{Contact criterion}

For the deformable surface at a single site, four distinct possible scenarios exist:

(i) The first possibility is a site that is already in contact and remains so. The old coordinate of the element is $u_{j+1}=z_{j+1}$. It evolves freely according to Eq. (18). Its new coordinate fulfills the requirement $u_{\mathrm{j}} \geqslant z_{\mathrm{j}}$ and hence stays in contact. The force at this site in contact is calculated according to Eq. (13). The coordinate of the deformable surface is set to the rigid one $u_{j}=z_{j}$.

(ii) The second possible result of the evolution of a site, which already has been in contact, is that it loses its contact $u_{k} \leqslant z_{k}$. The force acting at this site is set to zero, $f_{k}=0$.

(iii) Another outcome of the evolution is that a former free site hits the rigid surface and thus gets into contact $u_{j} \geqslant z_{j}$. Again the force $f_{j}$ in this newly established contact site is calculated in accordance with Eq. (13). Finally, the coordinate is set to the rigid one $u_{j}=z_{j}$.

(iv) The last possibility of evolution is a free contact that stays free. Its coordinate $u_{k}$ evolves according to the equation of motion Eq. (13) with external force set to zero and reference surface $u_{0 k}=0$. The solution of Eq. (18) $u_{k}$ gives the height of the surface at this site. There is no force acting between the surfaces at this site $f_{k}=0$.

In Fig. 3, a typical picture of the surfaces in contact can be found. We consider a displacement controlled process. In order to achieve a certain indention $d$, the coordinates of the rigid body is adjusted by an overall shift of this surface through this indentation

$$
z_{i}=z_{0 i}+d
$$

Summing the local forces $f_{i}$ over all sites yields the 


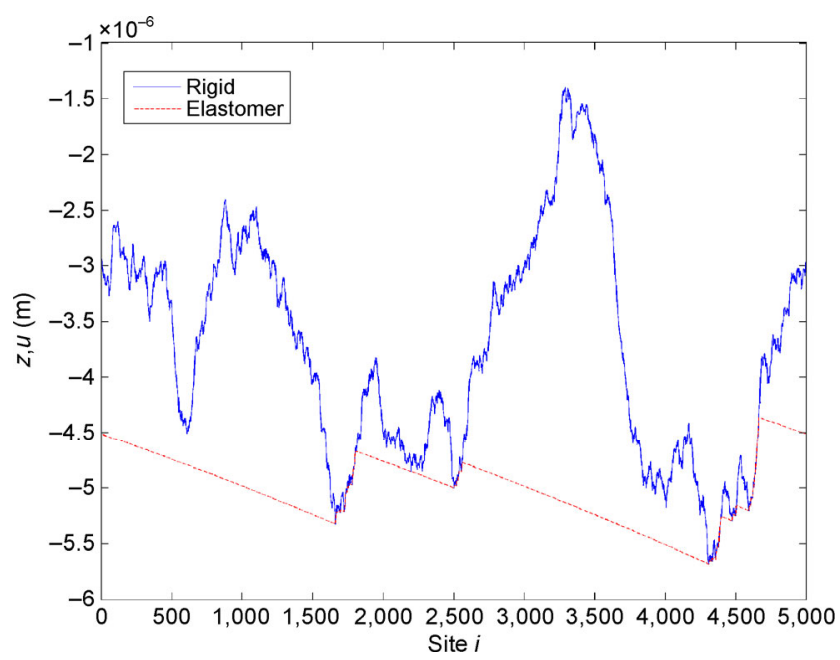

Fig. 3 Steady state configuration of the considered surfaces in contact: rigid rough surface (blue solid line) and deformed elastomer (red dashed line).

total force exerted between the surfaces. This force equals the normal forc $F_{\mathrm{N}}=\sum_{i} f_{i}$. For the computation of the frictional force $F_{x}$, the tangential force for all sites

$$
F_{x}=\left|\sum_{i} f_{i} \frac{\mathrm{d} z_{i}}{\mathrm{~d} x}\right|=\left|\sum_{i} f_{i} \frac{z_{i+1}-z_{i}}{\mathrm{~d} x}\right|
$$

is calculated. The coefficient of friction is defined as ratio between the total tangential and total normal force

$$
\mu=\frac{F_{x}}{F_{\mathrm{N}}}
$$

The contact length is computed as the sum over the number of contact site times the spatial step

$$
L_{\text {cont }}=\sum_{\text {cont }} \mathrm{d} x=\mathrm{d} x N_{\text {cont }}
$$

Another quantity of interest is the surface gradient in contact sites

$$
\nabla z_{\text {cont }}=\sqrt{\frac{1}{N_{\text {cont }}} \sum_{j}\left(\frac{z_{j+1}-z_{j}}{\mathrm{~d} x}\right)^{2}}
$$

which is a measure for the roughness that is actually experienced by the elastomer surface.

\subsection{Temperature dependence}

The temperature dependence of the simulated tribo- logical contact is included through a viscosity, which is considered to be thermally activated

$$
\eta(T)=\eta_{1} \exp \left[\frac{U_{0}}{k_{\mathrm{B}} T}\right]
$$

where $T$ is temperature, $U_{0}$ an activation potential, and $k_{\mathrm{B}}$ the Boltzmann constant. This influences the thermal behavior of the contact. For small deviations from a certain configuration $\left(\eta_{0}, T_{0}\right)$, the temperature dependence of the viscosity is expressed as

$$
\begin{aligned}
& \eta(T)=\eta_{0} \exp \left[\frac{U_{0}}{k_{\mathrm{B}}}\left(\frac{1}{T}-\frac{1}{T_{0}}\right)\right] \approx \eta_{0} \exp \left[-\frac{U_{0}}{k_{\mathrm{B}} T_{0}^{2}} \Delta T\right], \\
& \eta(\Delta T)=\eta_{0} \exp [-\alpha \Delta T]
\end{aligned}
$$

where $\Delta T=T-T_{0}$ and $\alpha \equiv U_{0} /\left(k_{\mathrm{B}} T_{0}^{2}\right)$. An increase of the temperature by $30 \mathrm{~K}$ typically halves the viscosity [23]. This corresponds to an activation energy $U_{0}$ of $11 \log 2=7.62$ in units of $k_{\mathrm{B}} T_{0}$ at $T_{0}=300 \mathrm{~K}$.

\subsection{Power dissipation}

The temperature change originates from the dissipated power in a viscoelastic material. The energy loss in the viscous part of the element leads to a rise in temperature in the contact. Simple considerations about the heat flow in the contact and bulk give rise to a temperature field in the contact. As shown in Ref. [20], the MDR concept gives a straightforward recipe to include heat transfer.

In the substitute MDR model [20, 24], the heat flow in a single element of the Winkler foundation is given by

$$
p=2 \lambda \Delta T \mathrm{~d} x
$$

for a thermal conductivity $\lambda$. Reversing this relation, we obtain for the temperature change in the onedimensional substitute model

$$
\Delta T_{i}=\frac{p_{i}}{2 \lambda \mathrm{d} x}
$$

For the Kelvin model in a one-dimensional Winkler foundation, the power dissipated in the element amounts to

$$
p_{i}=4 \eta_{i} \dot{u}_{i}^{2} \mathrm{~d} x
$$

Thus, for an element with thermal conductivity $\lambda$, 


$$
\Delta T_{j}=\frac{2 \eta_{j} \dot{u}_{j}^{2}}{\lambda}
$$

Keeping in mind that the shift in temperature causes a change in the thermal and relaxation behavior of the elastomer, we model it exclusively as a changing viscosity. For every site, the viscosity $\eta_{i}$ should be adapted to the temperature in this element. So we get a consistency equation at each site in contact

$$
\Delta T_{j}=2 \frac{\eta_{0} v_{0}^{2}}{\lambda}\left(\frac{\mathrm{d} z_{j}}{\mathrm{~d} x}\right)^{2} \exp \left[-\alpha \Delta T_{j}\right]
$$

For elements in contact the change of viscosity leads to a different force experienced by the surface.

\section{Results and discussion}

\subsection{Single asperity-conical indenter}

Since a rough surface may be seen as a collection of single asperities we review shortly the indentation of a cone into an elastomer (see Fig. 4). This set up is far easier to analyze since the associated temperature is constant for the entire contact zone. In this section, we consider a rigid cone with slope $c$ penetrating a viscoelastic medium with indentation depth $d$. The single conical indenter can be treated in a displacement controlled process: The indentation is fixed and the normal force $F_{\mathrm{N}}$ for this given indentation is calculated. A similar treatment for the force controlled process is found in Ref. [23]. The shape of the indenter is

$$
z=c|x|-d=g(x)-d
$$

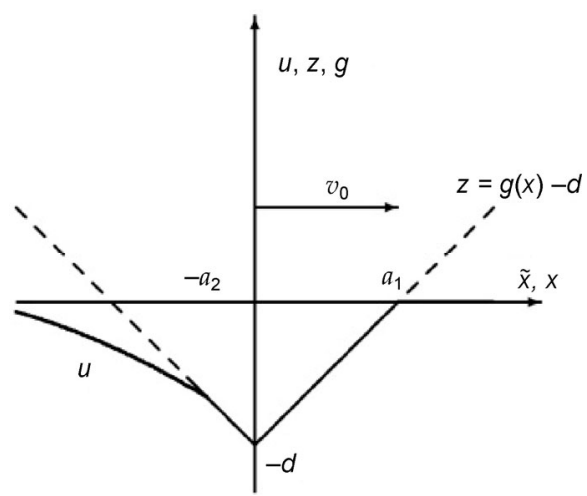

Fig. 4 Geometrical set up for a conical indenter.
Since the indenter is moving at constant speed $v_{0}$ and we follow with it, we denote the corresponding coordinate by $x=\tilde{x}-v_{0} t$. The viscoelastic medium is characterized as above by a parallel spring damper combination [20]

$$
k=4 G \mathrm{~d} x, \quad \theta=4 \eta \mathrm{d} x
$$

The start and end point of the contact region are called $-a_{2}$ and $a_{1}$. The vertical displacements $u$ in the contact can be found from the geometry

$$
u(x, t)=z(x)=-d+g\left(\tilde{x}-v_{0} t\right)
$$

The velocity of the elastomer surface in contact is then

$$
\frac{\mathrm{d} u}{\mathrm{~d} t}=\frac{\mathrm{d} g}{\mathrm{~d} t}=-v_{0} g^{\prime}(x)
$$

The surface exerts a force on a single element

$$
f_{\mathrm{N}}=4\left(G(-d+g(x))-\eta v_{0} g^{\prime}(x)\right) \mathrm{d} x
$$

The first contact point is determined by the condition that the surface is undisturbed, i.e.,

$$
u\left(a_{1}, t\right)=0
$$

The surfaces detach when the force in the element vanishes

$$
f_{\mathrm{N}}\left(-a_{2}, t\right)=0
$$

These requirements may be rewritten as

$$
a_{1}=d / c, \quad a_{2}=d / c-v_{0} \eta / G
$$

We introduce a dimensionless velocity

$$
\bar{v}_{c}=\frac{c v_{0} \eta}{G d}
$$

and consider two speed regimes. In the first range $\bar{v}_{c}<1$, the elastomer attaches to the indenter even after passing by the tip. In the second regime $\bar{v}_{c}>1$, the relaxation of the elastomer is so slow that it loses contact after the tip. In the first speed domain, the normal force between the indenter and the elastomer is

$$
F_{\mathrm{N}}=\int_{-a_{2}}^{a_{1}} f_{\mathrm{N}} \mathrm{d} x=-\frac{2 G d^{2}}{c}\left(2+\bar{v}_{c}^{2}\right)
$$


The frictional force is given by the tangential force. Hence,

$$
F_{x}=\int_{-a_{2}}^{a_{1}} f_{\mathrm{N}} g^{\prime}(x) \mathrm{d} x=-2 G d^{2}\left(4 \bar{v}_{c}-\bar{v}_{c}^{2}\right)
$$

The coefficient of friction thus becomes

$$
\frac{\mu}{c}=\frac{4 \bar{v}_{c}-\bar{v}_{c}^{2}}{2+\bar{v}_{c}^{2}} \quad \bar{v}_{c}<1
$$

Looking at higher velocities $\bar{v}_{c}>1$, the contact region ends at the tip, $a_{2}=0$. Hence, the integration limits have to be adapted properly

$$
\begin{aligned}
& F_{\mathrm{N}}=\int_{0}^{a_{1}} f_{N} \mathrm{~d} x=\frac{2 G d^{2}}{c}\left(1+2 \bar{v}_{c}\right), \\
& F_{x}=\left|\int_{0}^{a_{1}} f_{N} g^{\prime}(x) \mathrm{d} x\right|=2 G d^{2}\left(1+2 \bar{v}_{c}\right)
\end{aligned}
$$

The coefficient of friction thus becomes

$$
\frac{\mu}{c}=1 \quad \bar{v}_{c}>1
$$

Summarizing these results coefficient of friction in this picture is given in terms of the dimensionless velocity

$$
\frac{\mu}{c}= \begin{cases}\frac{4 \bar{v}_{c}-\bar{v}_{c}^{2}}{2+\bar{v}_{c}^{2}} & \bar{v}_{c}<1 \\ 1 & \bar{v}_{c}>1\end{cases}
$$

The coefficient of friction for a conical indenter is described by a rather simple rational function of the normalized velocity up to normalized speed one. Thereafter it is unity.

In the next step, we want to include heat generation in the contact and the corresponding temperature. The power dissipation and hence the heat production in each element take place in the damper according to Eq. (29). As long as there is contact between the indenter and the elastomer the surface $u$ follows the rigid profile so that $\dot{u}= \pm c v_{0}$ for the conical indenter. Substituting this into Eq. (30) we learn that the temperature is shifted by

$$
\Delta T=2 \frac{\eta_{0} v_{0}^{2} c^{2}}{\lambda}
$$

for a temperature independent viscosity. Allowing for the elastomer to react on the temperature shift, i.e., introducing a temperature dependent viscosity $\eta(T)$, leads to a consistency equation Eq. (31)

$$
\Delta T=2 \frac{\eta_{0} v_{0}^{2} c^{2}}{\lambda} e^{-\alpha \Delta T}
$$

This temperature is the same for all sites in the contact as long as the slope is constant for the indenter.

We introduce a number of short hand notations for frequently appearing combinations of quantities

$$
\begin{aligned}
& \chi=\frac{2 \alpha G^{2} d^{2}}{\lambda \eta_{0}}, \\
& \phi=\frac{2 \alpha \eta_{0} c^{2} v_{0}^{2}}{\lambda}=\chi \bar{v}_{c}^{2}, \\
& \xi=\alpha \Delta T=\frac{U_{0} \Delta T}{k_{\mathrm{B}} T_{0}^{2}}
\end{aligned}
$$

$\xi$ provides a dimensionless temperature. In these variables, the consistency equation for the temperature has to be fulfilled

$$
\xi=\phi e^{-\xi}=\chi \bar{v}_{c}^{2} e^{-\xi\left(\chi, \bar{v}_{c}\right)}
$$

$\chi=0$ corresponds to a temperature independent viscosity. Figure 5 presents the numerical solution $\xi^{*}(\phi)$ of Eq. (50) and analytical approximations for small and large values of $\phi$. The solutions $\xi^{*}=\xi\left(\chi, \bar{v}_{c}\right)$ as functions of the parameter $\chi$ are plotted in Fig. 6 .

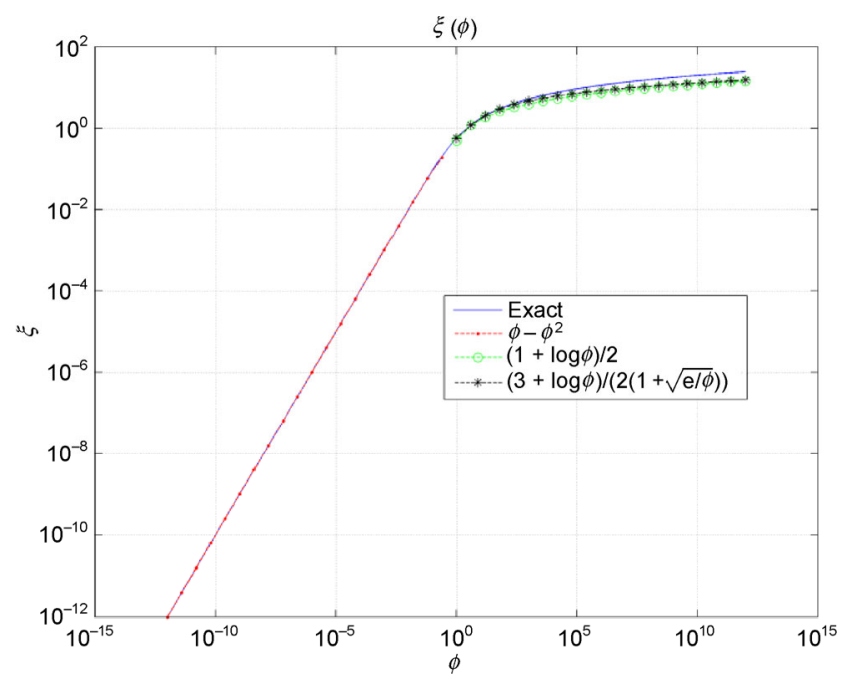

Fig. 5 Numerical solution $\xi^{*}$ as function of $\phi$ (blue solid). Analytical approximations for large (green dashed-circle, black dashed-star) and small (red dash-dotted) values of $\phi$. 


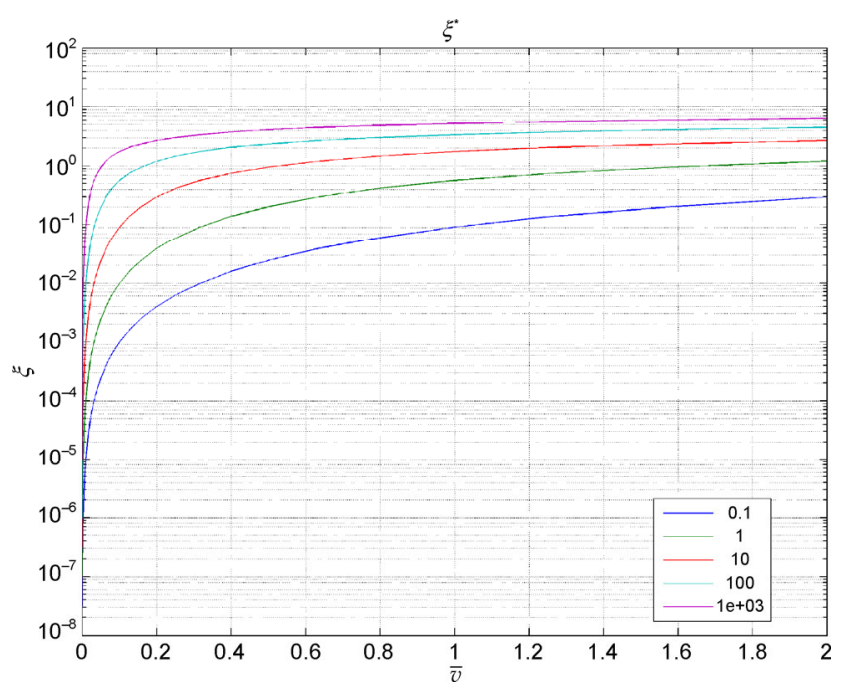

Fig. 6 Solution $\xi^{*}$ as function of $\bar{v}_{c}$ for different values of $\chi$.

Since the derivation of coefficient of friction Eq. (46) goes unaltered through, the normalized velocity is replaced by its temperature dependent analogue

$$
\bar{v}_{c} \rightarrow \tilde{v}_{c}=\bar{v}_{c} e^{-\xi^{*}}=\bar{v}_{c} e^{-\alpha \Delta T}
$$

Figure 7 provides a plot of the two different velocities. The corrected coefficient of friction is expressed as

$$
\frac{\mu}{c}= \begin{cases}\frac{4 \tilde{v}_{c}-\tilde{v}_{c}^{2}}{2+\tilde{v}_{c}^{2}} & \tilde{v}_{c}<1 \\ 1 & \tilde{v}_{c}>1\end{cases}
$$

It should be emphasized that $\tilde{v}_{c}$ should never exceed one for the coefficient of friction to remain at less than unity. This also leads to a critical value for the parameter $\chi$. From Eqs. (50) and (51), one can deduct that at the solution $\xi^{*}=1$ the maximum is reached. Simultaneously the speed at the extreme is given by $\bar{v}_{\mathrm{E}}=\sqrt{\exp \left(-\xi^{*}\right) / \chi}$. Hence, the critical value of $\chi_{c}=1 / e$ divides the behavior of the coefficient of friction into two regions (Fig. 8). For value below the critical value the coefficient of friction may rise to the saturation level and hit unity. Above the critical value, the speed $\tilde{v}_{c}$ never exceeds unity and the coefficient of friction never reaches unity. A comparison between the simulation of such a contact and the analytical formula Eq. (52) is displayed in Fig. 9. The agreement is good for a large range of dimensionless velocities. Numerical deviations are due the fact that for very small indentations the number of contacts is very small.

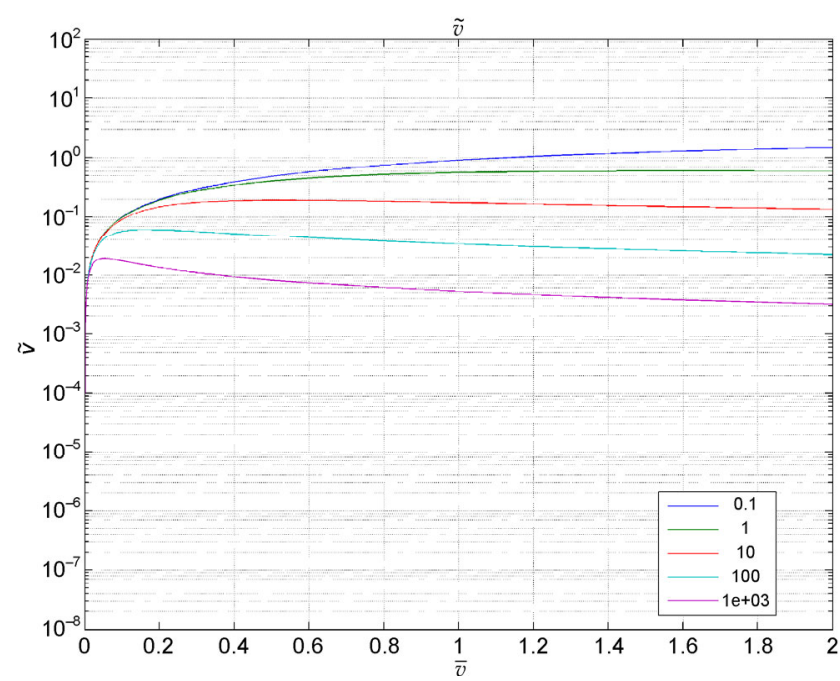

Fig. 7 Temperature corrected velocity $\tilde{v}_{c}$ as function of $\bar{v}_{c}$ for different values of $\chi$.

In the other extreme, there might be full contact over the entire simulation range.

$\bar{v}_{c}$ fulfills the consistency equation Eq. (50) Hence,

$$
\bar{v}_{c}=\sqrt{\frac{\alpha \Delta T}{\chi}} e^{\alpha \Delta T / 2}=\sqrt{\beta \Delta T} e^{\alpha \Delta T / 2}
$$

Substituting this into Eq. (52), we find for the temperature dependence of the coefficient of friction for the rigid cone shift in the contact

$$
\frac{\mu(\Delta T)}{c}= \begin{cases}\frac{4 \sqrt{\beta \Delta T} e^{-\alpha \Delta T / 2}-\beta \Delta T e^{-\alpha \Delta T}}{2+\beta \Delta T e^{-\alpha \Delta T}} & \beta \Delta T e^{-\alpha \Delta T}<1 \\ 1 & \beta \Delta T e^{-\alpha \Delta T}>1\end{cases}
$$

with $\beta \equiv \alpha / \chi=\lambda \eta_{0} /\left(2 d^{2} G^{2}\right)$ as a further inverted temperature. Note that $\beta$ is independent of $c$, i.e., the slope is not involved here. Neither, there is a dependence on $v_{0}$.

Figure 10 shows a comparison between the numerical simulation of a cone and the analytical expression Eq. (54). The difference between the expression Eq. (52) and Eq. (54) lies in the fact that the first one describes the coefficient of friction in a system with speed and indentation. Given these two quantities the coefficient of friction and the temperature are calculated. In the latter parametrization, the frictional process is parametrized by the indentation and the temperature. Hence, we have given expressions for the coefficient of friction for a conical single asperity as a function of indentation, normalized velocity, or temperature. 


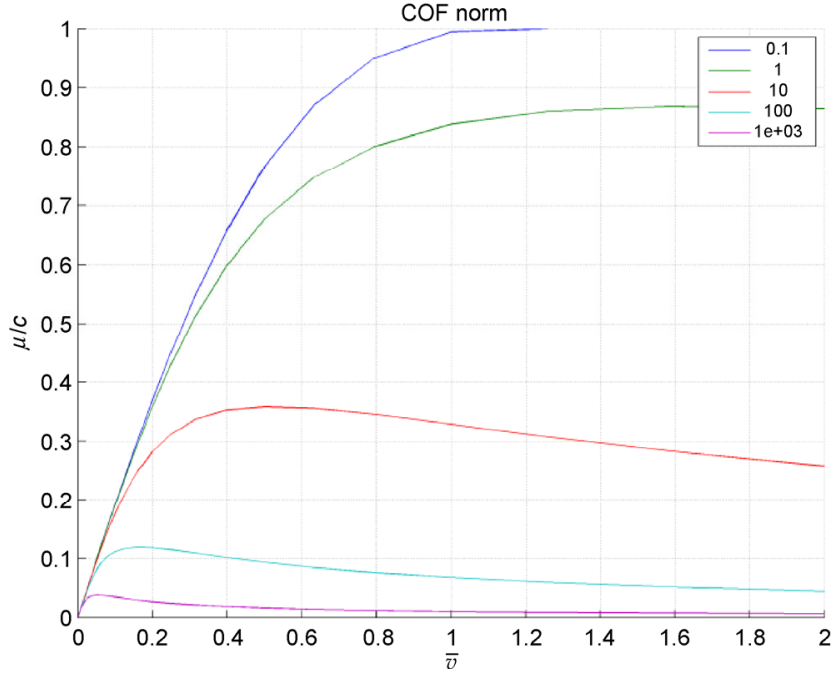

Fig. 8 Normalized coefficient of friction $\mu / c$ as function of $\bar{v}_{c}$ for different values of $\chi$. For values of $\chi>\chi_{c}=1 / e=0.367$ the coefficient of friction never reaches unity.

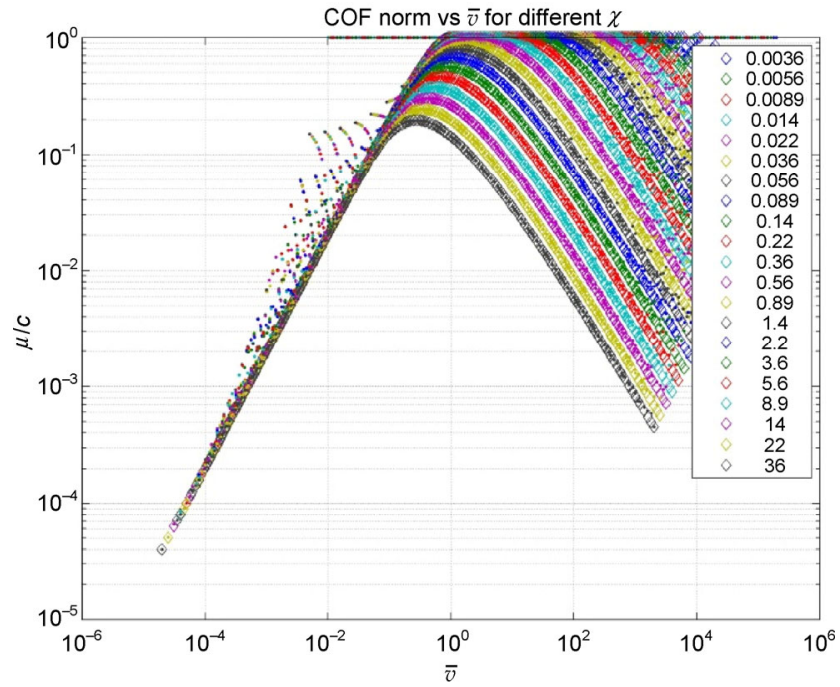

Fig. 9 Normalized coefficient of friction $\mu / c$ as function of $\bar{v}_{c}$ for different values of $\chi$. Numerical simulation (dots) and analytical estimate Eq. (52) (diamonds). Again, for values of $\chi>\chi_{c}=1 / e=0.367$ the coefficient of friction never reaches unity.

\subsection{Rough surface}

Turning back to our original problem, we want to explore the frictional behavior of an elastomer in contact with a rough surface. The rough surface is characterized by Eq. (5). Thus, by construction the RMS slope is

$$
\nabla z=2 \pi \frac{h}{L}\left(\frac{N}{2}\right)^{1-H} \sqrt{\frac{H}{1-H}}=\pi \frac{h}{\mathrm{~d} x}\left(\frac{2 \mathrm{~d} x}{L}\right)^{H} \sqrt{\frac{H}{1-H}}
$$

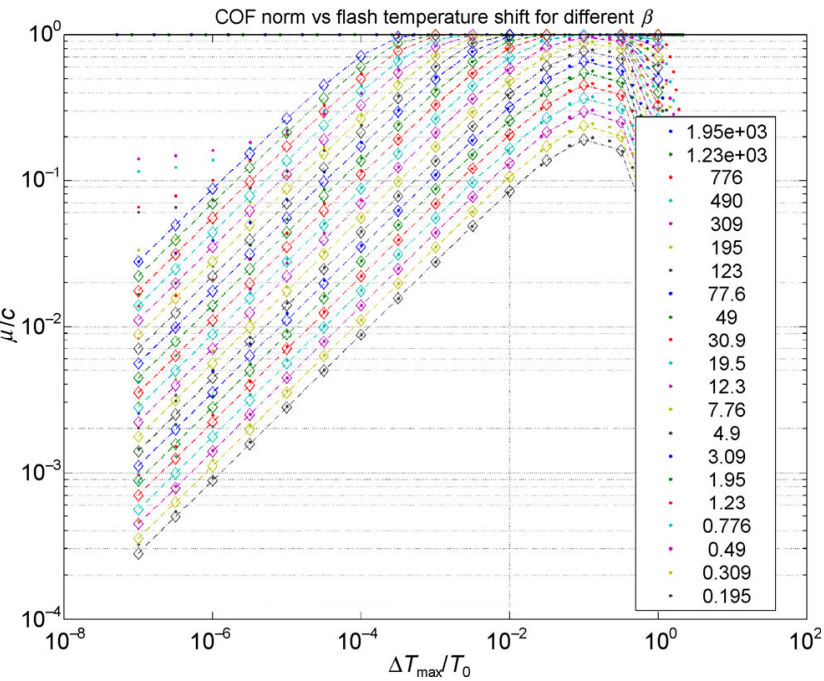

Fig. 10 Normalized coefficient of friction as a function of the temperature shift for different values of the parameter $\beta$. The numerical simulations (dots) fit well with the analytic estimate Eq. (54) (diamonds).

The rigid surface before indentation $z_{0}$ is normally distributed with vanishing mean and standard deviation $\sigma=h$ by construction. Its derivative is a normally distributed random variable as well. Its mean is again vanishing but its standard deviation $\nabla z$ depends on $H$ as one can see in Eq. (55). This rigid surface is brought into contact with an elastomer in a displacement controlled process. First the properties of the elastomer are considered to be temperature independent, later on the viscosity will be thermally activated.

\subsection{Numerical values}

Since we consider a randomly rough surface the results shown in the following base on 200 different realizations of each surface with a length of $L=0.01 \mathrm{~m}$ divided into $N=5,000$ elements. The roughness $h$ of the surface was chosen to be $0.5 \mu \mathrm{m}$. The Hurst exponents are chosen in the range of $[0,1]$. The elastomer is characterized by a modulus $G=10 \mathrm{MPa}$ and a viscosity $\eta_{0}=10^{4} \mathrm{~Pa} \cdot \mathrm{s}$. The heat flow is controlled by a specific thermal conductivity of $\lambda=0.013 \mathrm{~W} /(\mathrm{m} \cdot \mathrm{K})$. The velocities lay in the range from $2 \mu \mathrm{m} / \mathrm{s}$ up to $20 \mathrm{~m} / \mathrm{s}$.

\subsection{Displacement controlled process-constant viscosity}

In a displacement controlled system, the contact configuration depends on the robust external quantity 
indentation depth $d$. Figure 11 shows a typical picture of the coefficient of friction as a function of indentation and speed. There exists a saturation plateau for sufficiently large indentations and velocities. Besides in a region of small velocities there is a linear dependence. Up to a value of about the roughness $h$ the indentation does not play a role but for larger indentations the coefficient of friction declines with larger indentation. We want to find an estimate to express this behavior. The normal force

$$
F_{\mathrm{N}}=4 G \mathrm{~d} x \sum_{\text {cont }}\left(d-z_{0 i}\right)+4 \mathrm{~d} x v_{0} \sum_{\text {cont }} \eta_{j} \frac{\mathrm{d} z_{j}}{\mathrm{~d} x}
$$

and the coefficient of friction

$$
\begin{gathered}
\mu=\frac{F_{\mathrm{t}}}{F_{\mathrm{N}}}=\frac{4 G \mathrm{~d} x \sum_{\text {cont }}\left(d-z_{0 j}\right) \frac{\mathrm{d} z_{j}}{\mathrm{~d} x}+4 \mathrm{~d} x v_{0} \sum_{\text {cont }} \eta_{j}\left(\frac{\mathrm{d} z_{j}}{\mathrm{~d} x}\right)^{2}}{4 G \mathrm{~d} x \sum_{\text {cont }}\left(d-z_{0 j}\right)+4 \mathrm{~d} x v_{0} \sum_{\text {cont }} \eta_{j} \frac{\mathrm{d} z_{j}}{\mathrm{~d} x}}= \\
\frac{\frac{d}{h} \frac{1}{N} \sum_{\text {cont }} \frac{\mathrm{d} z_{j}}{\mathrm{~d} x}-\frac{1}{N} \sum_{\text {cont }} \frac{z_{0 j}}{h} \frac{\mathrm{d} z_{j}}{\mathrm{~d} x}+\frac{1}{N} \frac{\eta v_{0}}{G h} \sum_{\text {cont }}\left(\frac{\mathrm{d} z_{j}}{\mathrm{~d} x}\right)^{2}}{\frac{d}{h} \frac{L_{\text {cont }}}{L}-\frac{1}{N} \sum_{\text {cont }} \frac{z_{0 j}}{h}+\frac{1}{N} \frac{\eta v_{0}}{G h} \sum_{\text {cont }} \frac{\mathrm{d} z_{j}}{\mathrm{~d} x}}
\end{gathered}
$$

depend on the contact figuration through the sums. We assume constant viscosity for all elements for the moment. For rather high velocities, the last terms in nominator and denominator control the system.

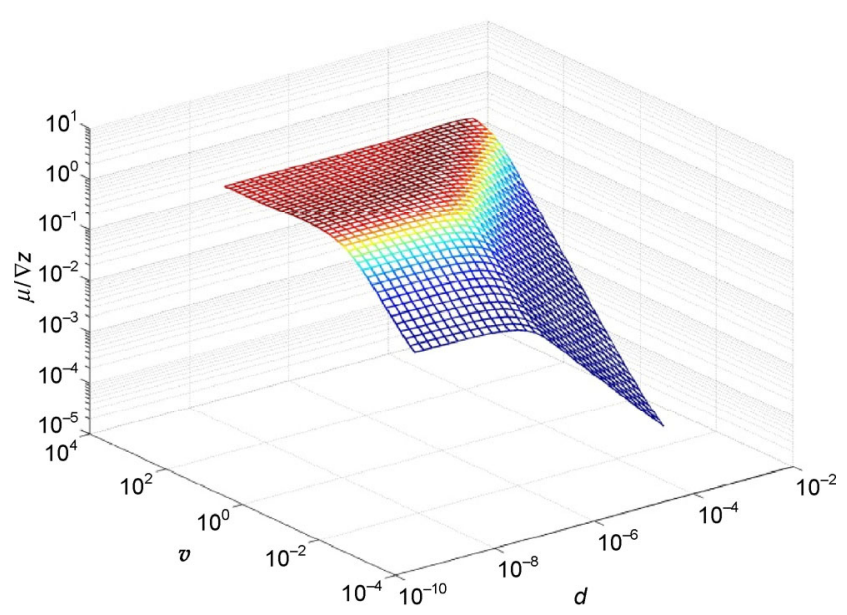

Fig. 11 Normalized coefficient of friction as function of indentation $d$ and velocity $v$ for surfaces with $H=0.7$. There is a plateau region for sufficiently large speeds and small indentations.
In this pure viscous regime, the coefficient of friction becomes independent of speed

$$
\mu=\frac{\frac{\eta v_{0}}{G h} \frac{1}{N} \sum_{\text {cont }}\left(\frac{\mathrm{d} z_{j}}{\mathrm{~d} x}\right)^{2}}{\frac{\eta v_{0}}{G h} \frac{1}{N} \sum_{\text {cont }} \frac{\mathrm{d} z_{j}}{\mathrm{~d} x}}=\frac{\sum_{\text {cont }}\left(\frac{\mathrm{d} z_{j}}{\mathrm{~d} x}\right)^{2}}{\sum_{\text {cont }} \frac{\mathrm{d} z_{j}}{\mathrm{~d} x}}
$$

Here, the plateau region is reached where most of elastomer materials are used. The nominator is nothing but $N_{\text {cont }} \nabla z_{\text {cont }}^{2}$. The actual contact configuration determines the quotient. If one assumes that half of the surface is in contact the coefficient of friction is approximated by $\mu=\sqrt{\pi / 2} \nabla z$ but in the case of high speed much less than half of the elements are in contact. Rather the elastomer detaches from the rigid surface at the back of an asperity and does not reattach. The deformed surface stays at about the same height due to the high velocity until it hits the next asperity and is further deformed. The elastomer sees only one-sided contacts. The maximal values of the coefficient of friction are dependent on the contact configuration. In Fig. 12, the maximal value of the normalized coefficient of friction is displayed for surfaces with different Hurst exponents and indentations. The maxima are fairly good approximated by

$$
\mu=\sqrt{2} \nabla z_{\text {cont }}
$$

in the plateau region.

Another region of interest is entered when only small indentations on the roughness scale are to be found $(d \ll h)$ at small velocities. The elastomer has time to relax back towards its original flat shape. The indentation is so small that it does not alter this behavior. Hence we find a partial contact of the elastomer at the asperities which lay underneath the undeformed surface. The terms with the indentation $d$ are neglected. The coordinates of the rough surface are of the order of magnitude $h$.

For small velocities, we see

$$
\mu=\frac{-\sum_{\text {cont }} \frac{z_{0 i}}{h} \frac{\mathrm{d} z_{j}}{\mathrm{~d} x}+\frac{\eta v_{0}}{G h} \sum_{\text {cont }}\left(\frac{\mathrm{d} z_{j}}{\mathrm{~d} x}\right)^{2}}{\sum_{\text {cont }} \frac{z_{0 i}}{h}}
$$




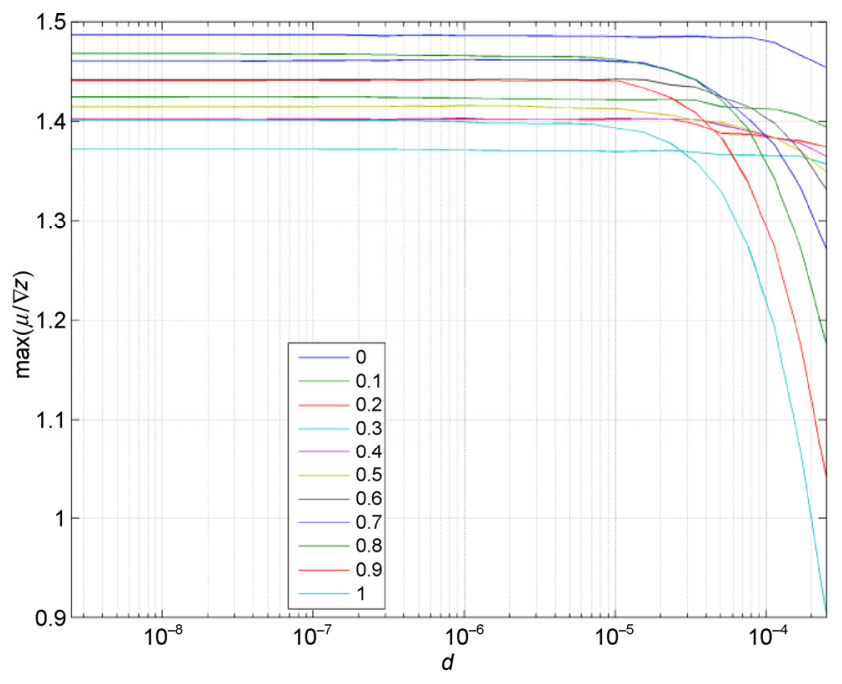

Fig. 12 Maxima of the normalized coefficient of friction as function of indentation $d$ for surfaces with different Hurst exponents. The maximal value is fairly constant at a value of about $\sqrt{2}$ as long as the indentation is less than the roughness, which is the region of interest in the plateau regime.

The first term may be rewritten as the difference of the square of the coordinates at the start and end of each contact. The order of magnitude of $z_{0}$ is $h$. In our simulations, it vanishes as it should. Summarizing this, we find

$$
\mu \approx \frac{\eta v_{0}}{G \sum_{\text {cont }} z_{0 i}} N_{\text {cont }} \nabla z_{\text {cont }}^{2} \approx \frac{\eta v_{0}}{G h} \nabla z_{\text {cont }}^{2}
$$

The last approximation takes the order of magnitude of the profile into account.

On the other hand for large indentations $(d>>h)$ and small velocities we find nearly full contact. The elastomer has time to relax and get into contact with the rough surface. The rough surface is lowered to the mean value $d$ and hence many elements get into contact. The coefficient of friction can then be approximated by

$$
\mu \approx \frac{\frac{d}{h} \sum_{\text {cont }} \frac{\mathrm{d} z_{j}}{\mathrm{~d} x}+\frac{1}{N} \frac{\eta v_{0}}{G h} \sum_{\text {cont }}\left(\frac{\mathrm{d} z_{j}}{\mathrm{~d} x}\right)^{2}}{\frac{d}{h} \frac{L_{\text {cont }}}{L}} \approx \frac{\eta v_{0}}{G d} \nabla z_{\text {cont }}^{2}
$$

since the first term just measures the difference between the first and last coordinate and vanishes.

Interpolating between these regions results in an estimate for the coefficient of friction

$$
\mu=\left[\left(\sqrt{2} \nabla z_{\text {cont }}\right)^{-2}+\left(\frac{\eta v_{0}}{G h} \nabla z_{\text {cont }}^{2}\right)^{-2}+\left(\frac{\eta v_{0}}{G d} \nabla z_{\text {cont }}^{2}\right)^{-2}\right]^{-1 / 2}
$$

or approximately

$$
\frac{\mu}{\nabla z}=\left[(1.4)^{-2}+\left(1.15 \bar{v}_{H}\right)^{-2}+\left(\frac{\bar{v}_{H}}{\bar{d}}\right)^{-2}\right]^{-1 / 2}
$$

where $\bar{d}=d / h$ and $\bar{v}_{H}=\eta_{0} v_{0} \nabla z /(G h)$. The empirical values give best fit to the numerical simulations. In Fig. 13, a comparison between the numerical simulation and the estimate Eq. (64) is shown. The estimate is a very good approximation over a large range of values of the dimensionless velocity $\bar{v}_{H}$. Especially, the most interesting plateau region but even the small velocity range is in good agreement. For very small velocities, the numerical results show a tendency to a constant coefficient of friction. This is rather a numerical than a physical effect.

\subsection{Temperature dependence}

So far we considered a temperature independent viscosity. As in the case of the rigid cone we introduce a thermally activated viscosity according to Eq. (25). For the rough surface, there is yet another difference to the geometrically well-behaved cone. We cannot claim a constant and equal temperature in the elements of contact any longer. Rather, there is a certain temperature associated with every element in contact. In Fig. 14, we display the change in the behavior of the normalized coefficient of friction as a reaction to temperature dependent viscosity. First, the plateau at sufficiently high velocities is reached as in the temperature independent case. For even higher velocities, more power is available at the contact sites and temperature rises in contacting elements. This in turn leads to a lower viscosity. The elastomer gets more liquid and fills non-contact regions better. The dimensionless velocities drop exponentially as well.

\subsection{Thermal length}

Starting from the expression in the consistency equation 


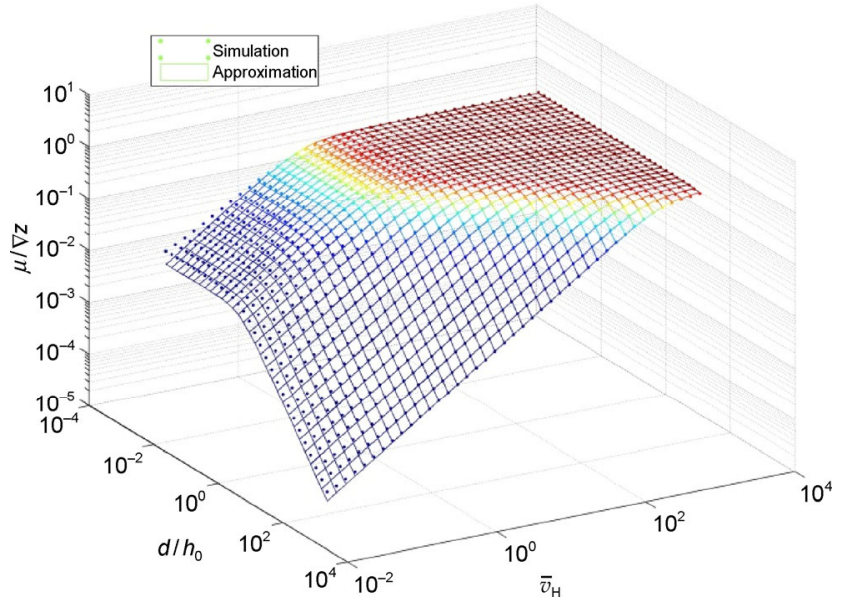

Fig. 13 Normalized coefficient of friction as function of velocity $\bar{v}_{H}$ for surfaces with $H=0.7$. The dots are results from numerical simulation. The solid lines are the estimate Eq. (64). The different horizontal lines correspond to different values of the normalized indentation $\bar{d} \in[0.005,500]$. For values less than unity they tend to collapse on the same line and switch from linear growth to the constant value $\sqrt{2}$ at about $\bar{v}_{H}=1$. For values well above unity the curves are well separated and hit the constant regime when $\bar{v}_{H}$ and $\bar{d}$ coincide.

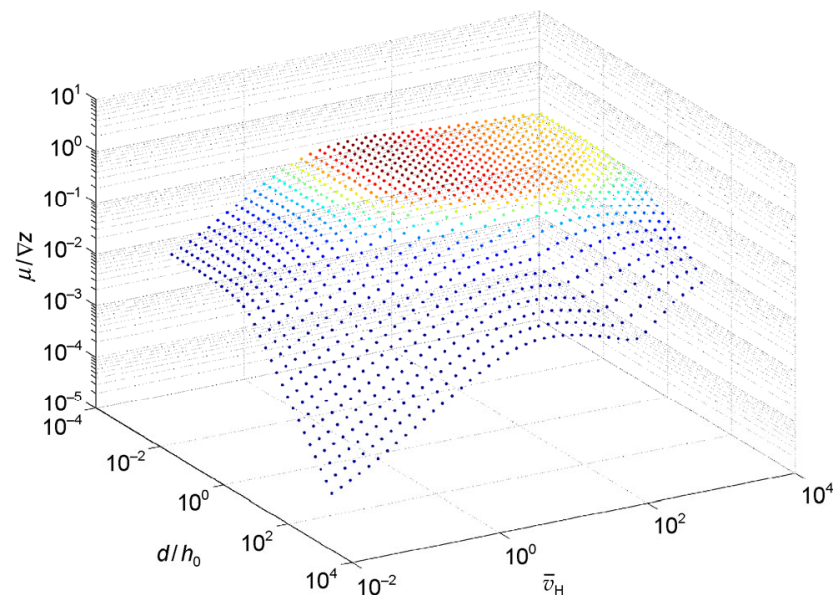

Fig. 14 Normalized coefficient of friction as function of indentation $\bar{d}$ and velocity $\bar{v}_{H}$ for surfaces with $H=0.7$ with temperature dependent viscosity. The coefficient of friction drops from its maximal value for further rising velocities and indentations larger than the thermal length compared to Fig. 11 since a more power in the contact leads to a rising temperature and an exponentially falling viscosity.

Eq. (50) one can consider the dimensionless variable $\phi$ as the ratio between the relaxation length scale set by $v_{0} \eta_{0} / G$ and another length scale set by the thermal properties of the elastomer. It is set through the combination

$$
l_{\mathrm{t}}=\sqrt{\frac{\lambda \eta_{0}}{2 \alpha G^{2}}}
$$

We call this quantity thermal length. It only contains properties connected to the elastomer. Using this thermal length the parameter $\phi_{j}$ may be rewritten as a product of squares of a dimensionless velocity and of a ratio of length scale

$$
\phi_{j}=\bar{v}_{H j}^{2} \chi=\left(\frac{v_{0} \eta_{0}}{G h} \frac{\mathrm{d} z_{j}}{\mathrm{~d} x}\right)^{2}\left(\frac{h}{l_{\mathrm{t}}}\right)^{2}
$$

Here we used the idea that the rough surface may be interpreted as a collection of asperities. The slope $c$ is replaced by the slope between the elements in contact $\mathrm{d} z_{j} / \mathrm{d} x$.

The indentation for a single cone $d$ is comparable to the height difference between two subsequent elements, which is of the order of the roughness $h$. The solution of the consistency equation depends on the thermal length scale. Especially, the velocity is altered through the exponential factor. In practice this means that the coefficient of friction diminishes when the length scale of motion becomes larger than the thermal length scale (see Fig. 14). With the values chosen in our calculation the thermal length is about $10 h$. As expected the coefficient of friction drops from its maximal value for indentations larger than $10 h$. The coefficient of friction for the temperature dependent case is

$$
\begin{gathered}
\mu=\frac{F_{\mathrm{t}}}{F_{\mathrm{N}}}=\frac{4 G \mathrm{~d} x \sum_{\text {cont }}\left(d-z_{0 i}\right) \frac{\mathrm{d} z_{j}}{\mathrm{~d} x}+4 \mathrm{~d} x v_{0} \sum_{\text {cont }} \eta_{j}\left(\frac{\mathrm{d} z_{j}}{\mathrm{~d} x}\right)^{2}}{4 G \mathrm{~d} x \sum_{\text {cont }}\left(d-z_{0 i}\right)+4 \mathrm{~d} x v_{0} \sum_{\text {cont }} \eta_{j} \frac{\mathrm{d} z_{j}}{\mathrm{~d} x}}= \\
\frac{\frac{d}{h} \frac{1}{N_{\text {cont }}} \sum_{\text {cont }} \frac{\mathrm{d} z_{j}}{\mathrm{~d} x}-\frac{1}{N_{\text {cont }}} \sum_{\text {cont }} \frac{z_{0 i}}{h} \frac{\mathrm{d} z_{j}}{\mathrm{~d} x}+\frac{\eta v_{0}}{G h} \frac{1}{N_{\text {cont }}} \sum_{\text {cont }} e^{-\xi_{j}}\left(\frac{\mathrm{d} z_{j}}{\mathrm{~d} x}\right)^{2}}{\frac{d}{h}-\frac{1}{N_{\text {cont }}} \sum_{\text {cont }} \frac{z_{0 i}}{h}+\frac{\eta v_{0}}{G h} \frac{1}{N_{\text {cont cont }}} \sum^{-\xi_{j}} \frac{\mathrm{d} z_{j}}{\mathrm{~d} x}}
\end{gathered}
$$

where $\xi_{j}^{*}$ is the solution to the consistency equation

$$
\begin{aligned}
\xi_{j} & =\phi_{j} e^{-\xi_{j}}=\chi \bar{v}_{H j}^{2} e^{-\xi_{j}}, \\
\bar{v}_{H j} & =\frac{v_{0} \eta_{0}}{G h} \frac{\mathrm{d} z_{j}}{\mathrm{~d} x}
\end{aligned}
$$

as before (see Fig. 5). The elastic contributions to the 
normal and tangential forces are not altered through the temperature dependent viscosity. Hence, the coefficient of friction is changed only in those parameter ranges where the viscous parts play an important role. For small velocities, we see that the behavior of the coefficient of friction is scarcely changed. More interesting is the plateau region. The maximal value of the coefficient of friction is about 1.16. The plateau is not reached for large indentations when they reach the thermal length scale. Then the coefficient exhibits the behavior already shown in Fig. 9 for a single cone. The coefficient decreases. For even higher velocities the coefficient increases again but never reaches the plateau region. As a crude estimate for the coefficient of friction we propose

$$
\frac{\mu}{\nabla z}=\left[(1.16)^{-2}+\left(1.15 \tilde{v}_{H}\right)^{-2}+\left(\frac{\tilde{v}_{H}}{\bar{d}}\right)^{-2}\right]^{-1 / 2}
$$

where $\tilde{v}_{H}=\bar{v}_{H} e^{-\xi^{*}}$ is the corrected temperature dependent velocity. The consistency equation solution is found numerically for the RMS slope $\nabla z$. From this it becomes clear that the more the actual contact configuration departs from full contact the less reliable is the estimate. A comparison between numerical solution and estimate is displayed in Fig. 15.

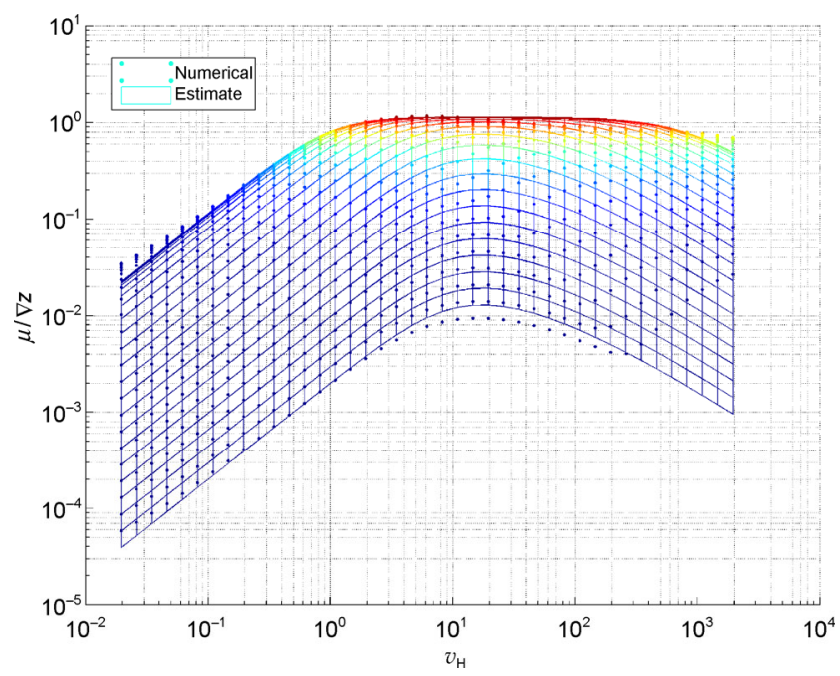

Fig. 15 Normalized coefficient of friction as function of indentation $\bar{d}$ and velocity $\bar{v}_{H}$ for surfaces with $H=0.7$ with temperature dependent viscosity and an estimate according to Eq. (69). For small indentations the curves collapse onto a single line (most upper part). For small indentations the maximal value is never reached which is also seen in the estimate. The drift to higher values at high speed and large indentation is not captured.

\subsection{Master curves}

We find a dependence of the coefficient of friction on the identified dimensionless parameters as follows

$$
\mu=\nabla z(\bar{d}) \Xi\left(\frac{\tilde{v}_{H}}{f(\bar{d})}\right)
$$

for a temperature dependent viscosity. The RMS slope in the contact region is governed by the indentation depth $d$ through the actual contact configuration. Another function $\Xi$ whose argument is a ratio between the normalized velocity and a function of the normalized indentation describes the dependence on the velocity. In a double logarithmic diagram of the coefficient of friction, curves as function of velocity will be shifted by some $\log \nabla z(\bar{d})$ vertically and $\log \left(\eta_{0} \nabla z\right)-\log (G f(\bar{d}))-\xi$ horizontally. This allows constructing master curves for elastomer properties that would be out of experimental reach. Similar conclusions are drawn in Ref. [26].

\section{Conclusions and outlook}

In this study, we have shown that the coefficient of friction between a rough rigid surface and a Kelvin model elastomer surface with Arrhenius-like temperature dependent viscosity is a function of the dimensionless combinations of material and geometric parameter. These combinations are dimensionless velocity $\bar{v}_{H}=v_{0} \eta_{0} \nabla z /(G h)$, indentation $\bar{d}=d / h$ and $\chi=2 \alpha G^{2} h^{2} /\left(\lambda \eta_{0}\right)$. It sets a thermal length scale $l_{\mathrm{t}}$ via $\chi=h^{2} / l_{\mathrm{t}}^{2}$. The latter parameter is independent of the geometry of the rough surface encoded by $\nabla z$. Former studies of the frictional behavior between elastomers and rough plain surfaces [22] or rough surfaces without thermal effects [27] point to the conclusion that the characteristics are similar to single asperity contact, which is explored in a displacement controlled process in this work. The coefficient of friction is a function of the dimensionless combinations of the loading and material parameters. Estimates for the coefficient of friction have been given for both temperature independent and dependent viscosity. The actual coefficient of friction is dependent on the geometrical set up of the problem including the roughness and indentation through the contact configuration. We anticipate that our results provide a 
qualitative understanding of the thermal behavior of elastomer surfaces in contact with rigid surfaces. The employment of master curves in this context has been addressed. Further investigations are required.

\section{Acknowledgements}

The author thanks V. L. Popov for discussions. R. H. is supported by DFG project PO 810/12-2.

Open Access: The articles published in this journal are distributed under the terms of the Creative Commons Attribution 4.0 International License (http:// creativecommons.org/licenses/by/4.0/), which permits unrestricted use, distribution, and reproduction in any medium, provided you give appropriate credit to the original author(s) and the source, provide a link to the Creative Commons license, and indicate if changes were made.

\section{References}

[1] Bowden F P, Tabor D. The Friction and Lubrication of Solids. Oxford University Press, 1985.

[2] Greenwood J A, Tabor D. The friction of hard sliders on lubricated rubber: The importance of deformation losses. Proc Phys Soc 71: 989-1001 (1958)

[3] Grosch K A. The relation between the friction and viscoelastic properties of rubber. Proc R Soc 274: 21-39 (1963)

[4] Barquins M, Courtel R. Rubber friction and the rheology of viscoelastic contact. Wear 32: 133-150 (1975)

[5] Klüppel M, Heinrich G. Rubber friction on self-affine road track. Rubber Chem Technol 73: 578-606 (2000)

[6] Persson B N J. Theory of rubber friction and contact mechanics, J Chem Phys 115: 3840-3861 (2001)

[7] Lorenz B, Persson B N J, Fortunato G, Giustiniano M, Baldoni F. Rubber friction for tire tread compound on road surfaces. J Phys: Condens Matter 25: 095007 (2013)

[8] Popov V L, Dimaki A V. Using hierarchical memory to calculate friction force between fractal rough solid surface and elastomer with arbitrary linear rheological properties. Tech Phys Lett 37: 8-11 (2011)

[9] Popova E, Popov V L. The research works of Coulomb and Amontons and generalized laws of friction. Friction 3(2): 183-190 (2015)

[10] Schallamach A. The load dependence of rubber friction. Proc Phys Soc B 65: 657-661 (1952)
[11] Ben-David O, Fineberg J. Static friction coefficient is not a material constant. Phys Rev Lett 106: 254301 (2011)

[12] Otsuki M, Matsukawa H. Systematic breakdown of Amontons' law of friction for an elastic object locally obeying Amontons' law. Sci Rep 3: 1586 (2013)

[13] Rubinstein S M, Cohen G, Fineberg J. Detachment fronts and the onset of dynamic friction. Nature 430: 1005-1009 (2004)

[14] Ben-David O, Cohen G, Fineberg J. The dynamics of the onset of frictional slip. Science 330: 211-214 (2010)

[15] Amundsen D S, Scheibert J, Thögersen K, Trömborg J, Malthe-Sörenssen A. 1D model of precursors to frictional stick-slip motion allowing for robust comparison with experiments. Tribol Lett 45(2): 357-369 (2012)

[16] Heise R, Popov V L. Adhesive contribution to the coefficient of friction between rough surfaces. Tribol Lett 39(3): 247-250 (2010)

[17] Heß M. About Mapping of Some Three-dimensional Contact Problems to Systems with a Lower Spatial Dimensionality. Göttingen: Cullier Verlag, 2011.

[18] Heß M. On the reduction method of dimensionality: The exact mapping of axisymmetric contact problems with and without adhesion. Phys Mesomech 15: 264-269 (2012)

[19] Pohrt R, Popov V L, Filippov A E. Normal contact stiffness of elastic solids with fractal rough surfaces for one- and three-dimensional systems. Phys Rev E 86: 026710 (2012)

[20] Popov V L, Heß M. Method of Dimensionality Reduction in Contact and Friction. Berlin Heidelberg: Springer, 2015.

[21] Popov V L, Dimaki A, Psakhie S, Popov M. On the role of scales in contact mechanics and friction between elastomers and randomly rough self-affine surfaces. Sci Rep 5: 11139 (2015)

[22] Li Q, Popov M, Dimaki A, Filippov A E, Kürschner S, Popov V L. Friction between a viscoelastic body and a rigid surface with random self-affine roughness. Phys Rev Lett 111: 034301 (2013)

[23] Dimaki A V, Popov V L. Coefficient of friction between a rigid conical indenter and a model elastomer: Influence of local frictional heating. Phys Mesomech 17(5): 57-62 (2014)

[24] Heise R. Flash temperatures generated by friction of a viscoelastic body. Facta Universitatis 13(1): 47-65 (2015)

[25] Popov V L. Contact Mechanics and Friction, 1st Ed. Berlin Heidelberg: Springer, 2010.

[26] Popov V L. What does friction really depend on? (Robust governing parameters in contact mechanics and friction). Phys Mesomech 18 (4): 5-11 (2015)

[27] Popov V L, Voll L, Li Q, Chai Y S, Popov M. Generalized law of friction between elastomers and differently shaped rough bodies. Sci Rep 4: 03750 (2014) 


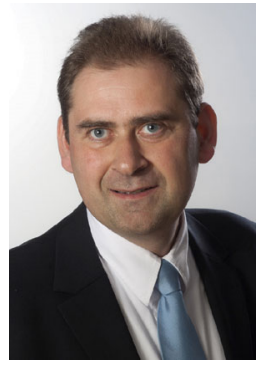

Rainer HEISE. He is postdoctoral researcher at Technische Universität Berlin. He studied engineering physics at Chalmers University of Technology in Göteborg (1996-2001) and received his $\mathrm{PhD}$ degree from Göteborg University in 2005. After a postdoc period at Albert Einstein
Institute in Potsdam Golm he works at the Department of System Dynamics and the Physics of Friction in the Institute of Mechanics at TU Berlin. His research interests include mathematical physics, numerical simulation of frictional processes, tribology, the influence of ultrasound on friction and issues related to materials such as elastomers but also particle physics. 